\title{
Is the progression rate of periodontitis related to subgingival biofilm composition or gingival crevicular fluid IL-1 $\beta$ and MMP-8 concentrations?
}

\author{
MAŁGORZATA NĘDZI-GÓRA, RENATA GÓRSKA, BARTŁOMIEJ GÓRSKI
}

Medical University of Warsaw, Warsaw, Poland

\begin{abstract}
Aim of the study: To analyze the composition of subgingival biofilm and to assess the concentration of IL-1 and MMP-8 in gingival crevicular fluid $(G C F)$ from deep periodontal pockets in patients with severe periodontitis to determine whether the presence of specific microbial species or the severity of the host's immune response can be helpful in assessing the dynamics of disease.

Material and methods: The study included 30 individuals with periodontitis Grade B and 19 subjects with periodontitis Grade C. Quantitative and qualitative microbiological analysis of flora in pockets $\geq 7 \mathrm{~mm}$ was performed for the presence of selected periopathogens of the orange, red complex and Aggregatibacter actinomycetemcomitans using real-time PCR. The concentrations of IL-1 and MMP-8 in GCF were evaluated with the ELISA method.

Results: There were no differences in the composition of the subgingival biofilm depending on the diagnosis. The concentration of MMP-8 in GCF was significantly higher in periodontitis Grade $C$ than in periodontitis Grade B (61 $\mathrm{ng} / \mu \mathrm{l}$ and $37 \mathrm{ng} / \mu \mathrm{l}$ respectively, $p=0.039)$. The concentration of IL-1 $\beta$ was similar in both groups. No significant correlations were observed between the occurrence of individual periopathogens and concentrations of MMP-8 and IL-1 $\beta$ depending on the diagnosis.

Conclusions: Periodontitis grade may not be distinguished according to microbial analysis of subgingival biofilm or to concentration of IL-1 $\beta$ in GCF. On the other hand, higher concentrations of $M M P-9$ in GCF from deep pockets may be helpful in detecting subjects particularly prone to occurrence and rapid progress of periodontitis.
\end{abstract}

Key words: periodontitis, markers, gingival crevicular fluid, microbe, mediators of inflammation.

(Cent Eur J Immunol 2020; 45 (4): 425-432)

\section{Introduction}

Periodontitis is both infectious and immunoinflammatory in its nature. Genetic susceptibility, by conditioning the immune-inflammatory response, determines the formation and course of periodontitis [1]. The 2017 World Workshop on the Classification of Periodontal and Peri-Implant Diseases and Conditions reiterated, that case definitions of periodontitis should be based on a combination of periodontitis stage and periodontitis grade [2]. Whereas staging relies on severity and extent of periodontitis, grading describes periodontitis progression rate and the relative risk of future tooth loss. The question of why the course and dynamics of periodontitis differ between individual patients is still not resolved. It has been implied, that genetic factors, besides microbe profile, influence the outcome and following progression of periodontitis in young patients, while determinants associated with lifestyle play more significant role later in life [3].

In 1998, Socransky et al. [4] chose 40 species of bacteria that were divided into seven complexes differing in the degree of periopathogenicity. Periopathogens occurring under dysbiosis conditions are located in the orange complex dominating in periodontal pockets 4-6 mm deep (e.g. Prevotella intermedia, Peptostreptococcus micros, Fusobacterium nucleatum, Eubacterium nodatum), while the most virulent microbiota associated with advanced periodontitis and pockets above $6 \mathrm{~mm}$ in the red complex (Porphyromonas gingivalis, Treponema denticola, Tannerella forsythia) and in the dark green complex (Aggregatibacter actinomycetemcomitans). Periopathogens show pathogenicity only in the case of host susceptibility

Correspondence: Bartłomiej Górski, Medical University of Warsaw, 18 Miodowa St., 00-246 Warsaw, Poland. e-mail: bartek_g3@tlen.pl Submitted: 5.04.2019; Accepted: 21.06.2019 
to colonization of pockets [5]. Pathogenic features of classical periopathogens have been quite well described. For example, $P$. gingivalis secretes large amounts of Porphyromonas peptidylarginine deiminase (PPAD), that neutralized human innate immune defenses at three specific levels (bacterial phagocytosis, capturing in NETs, and killing by the lysozyme-derived cationic antimicrobial peptide LP9). Moreover, $P$. gingivalis is able to produce a vast spectrum of gingipains that have a potential to degrade complement components (C3, C5), proinflammatory cytokines (IL-1, IL-6, IL-8) and immunoglobulins (IgA, IgM, IgG) [6]. T. denticola has a variety of virulence factors, such as production of proteolytic enzymes (oligopeptidase, dentipain, dentilisin etc.) [7]. T. forsythia secrets GroEL, which may synergize with IL-17 in bone resorption. On the other hand, A. actinomycetemcomitans is able to produce leukotoxin A, which binds to the lymphocyte function-associated antigen-1 (LFA-1) and induces $\beta$-hemolysis in red blood cells [8]. In addition to direct destruction of periodontal tissues by microbiota and inhibition of immuneinflammatory response, another mechanism of pathogenicity is the ability of periopathogens to penetrate into periodontal tissues $[9,10]$. In this way, the microbiota is protected against immune response.

Periopathogens in the subgingival biofilm are undoubtedly a primary etiological factor of periodontitis, but the formation and course of the disease are determined by genetically programmed immune response of the host [11]. The mechanism of bystander damage consists of secretion of excessive amounts of inflammatory agents and mediators in response to the presence of periopathogens. A number of bioactive agents have been suggested as potential biomarkers of the disease (macrophage inflammatory protein $[\mathrm{MIP}] 1 \alpha$, interleukin [IL] $1 \beta$, tumor necrosis factor [TNF] $\alpha$, matrix metalloproteinases [MMPs], IL-6). Neutrophils digest the pathogens through phagocytosis, damage the surroundings via superoxide anion production and release biologically active reagents, e.g. MMPs, which are proteases breaking down the connective tissue. One of the evaluated MMPs is MMP-8 (neutrophil collagenase), which degrades collagen, the main structural protein found in periodontal tissues. On the other hand, macrophages present antigens to the CD4+ cells ( $\mathrm{T}$ helper lymphocytes), secrete a handful of cytokines, and quite similarly to neutrophils, take part in phagocytosis. Cytokines are cell signaling molecules that regulate inflammatory and immune responses. One of the most important cytokines is IL-1 $\beta$, which stimulates the production of prostaglandin E2 in the bone, impedes osteoclast apoptosis and - through activating the receptor activator of nuclear factor kappa- $\beta$ ligand (RANKL) - prompts bone resorption [12]. IL-1 is secreted by different types of cells, such as osteoclasts and fibroblasts [35]. For these reasons an evaluation of the non-specific component of the immunological reaction is of great value.
Contemporary diagnostics, as well as forecasting of periodontitis, are based primarily on clinical and radiological parameters that allow to assess the severity of the disease. It is extremely difficult to estimate host's susceptibility to periodontitis. To cap it all, no indicators have been introduced so far, which could help detect a risk of future periodontitis at the subclinical level or estimate the progression rate and dynamics of the disease. For this reason, the search for unambiguous risk markers is still valid. The aim of the study was to analyze the composition of subgingival biofilm and concentration of IL-1 and MMP-8 in periodontal pockets $\geq 7 \mathrm{~mm}$ to verify whether presence of specific bacterial species or severity of the immune response of the host may be helpful in assessing periodontitis grading.

\section{Material and methods}

This study was performed at the Department of Periodontology and Oral Diseases of Medical University of Warsaw, Poland, after receiving a positive approval by the institutional review board (KB/171/2009). All clinical procedures were carried out in accordance with the Helsinki Declaration of 1975, as revised in Tokyo in 2004. Written informed consent forms were signed by every patient.

The study included 49 patients with Stage III periodontitis. The first group consisted of 30 individuals (17 females and 13 males with an average age of $49.0 \pm 13.1$ years) with Grade B periodontitis and the second group of 19 subjects (13 females and 6 males with an average age of $38.2 \pm 9.9$ years) with Grade C periodontitis. Diagnosis was given on the basis of a clinical and radiological examination in accordance with the applicable classification of periodontal diseases [2]. Periodontitis Stage III was diagnosed when: 1) interdental clinical attachment loss (CAL) $\geq 5 \mathrm{~mm}$; 2) radiographic bone loss extended to mid-third of root and beyond; 3 ) tooth loss due to periodontitis was $\leq 4$ teeth; 4) probing pocket depth (PPD) $\geq 6 \mathrm{~mm}$; 5) vertical bone loss $\geq 3 \mathrm{~mm}$. Periodontitis grades were evaluated indirectly (\% bone loss/age). Radiographic bone loss was evaluated on dental radiograms as percentage of root length divided by the age of the subject. Periodontitis Grade B - moderate rate of progression - was identified when $\%$ bone loss/age $=0.25$ to 1.0 , whereas periodontitis Grade $\mathrm{C}$ - rapid rate of progression - was recognized when $\%$ bone loss/age $>1.0$.

The criteria for inclusion in the study were 1) diagnosed advanced periodontitis stage III Grade B/C and 2) presence of at least one periodontal pocket $\geq 7 \mathrm{~mm}$ deep. The exclusion criteria were: 1 ) coexistence of a systemic condition that may affect the course of periodontal disease, 2) chronic intake of drugs that can modify the course of periodontal disease (antibiotics, steroids, anti-inflammatory drugs, immunosupressants, antiepileptic drugs and calcium channel blockers), 3) pregnancy/lactation, 
4) nicotinism (both active and past), 5) professional removal of deposits within 3 months before the examination, 6 ) topical use of products based on chlorhexidine within 3 months preceding the examination.

The study consisted of a clinical and laboratory part. Periodontal examination was carried out by a calibrated examiner who used a graded period probe (UNC probe $15 \mathrm{~mm}$, Hu-Friedy, Chicago, USA), and included: 1) a dichotomous (yes/no) FMPI index according to O'Leary et al. [14] on four tooth surfaces (i.e. distal, buccal, mesial, lingual). The index was determined by dividing the number of surfaces with plaque by the number of all tested surfaces; 2) dichotomous (yes/no) BoP index according to Ainamo and Bay [15]. Bleeding was assessed at six points for each tooth (i.e. distobuccal, buccal, mesiobuccal, distolingual, lingual, mesiolingual). The index was determined by dividing the number of bleeding points by the number of all examined points; 3) PPD was assessed at six points of each tooth as a distance from the gingival margin to the bottom of the pocket; 4) CAL was determined at six points of each tooth as a distance from the cemento-enamel junction (CEJ) to the bottom of the pocket; 5) the number of teeth present in the oral cavity.

Microbiological analysis was performed using readymade PET diagnostic kits from MIP Pharma (Icking, Germany). The deepest pocket $\geq 7 \mathrm{~mm}$ was selected for each patient. After isolating the examined pocket from the access of saliva, gingival plaque was removed from the tooth with a swab, then the examined area was dried. With sterile tweezers, one paper point was placed in the pocket. The points were placed fully into the pockets for 20 seconds, then packed into labeled test tubes and sent in transport packaging to the manufacturer's laboratory. If bleeding occurred or a paper point was contaminated with saliva, the procedure was rerun. With the use of real-time polymerase chain reaction (total-time PCR), the total number of bacteria in the sample was assessed, as well as the number and composition of nine periopathogens: four species of the orange complex (P. intermedia, P. micros, $F$. nucleatum, E. nodatum), three species of the red com- plex ( $P$. gingivalis, $T$. denticola, $T$. forsythia) and two species of the green complex (A. actinomycetemcomitans, Capnocytophaga gingivalis).

A sample of gingival crevicular fluid (GCF) was collected with a paper point (Paperstrip, OraFlow Inc., USA), that was inserted into the pocket for 30 seconds. Subsequently, paper points were inserted into a device assigned to measure the volume of the collected GCF (Periotron 8000 , OraFlow Inc., USA). Periotron was calibrated using standard volumes of human saliva $(0.25 \mu \mathrm{l} ; 0.75 \mu \mathrm{l}$; $1.0 \mu \mathrm{l} ; 1.25 \mu \mathrm{l})$ evaluated with a micropipette. Paper points were put into tubes containing phosphate-buffered saline, for IL-1 $\beta$ and MMP-8 $200 \mu \mathrm{l}$ and $500 \mu \mathrm{l}$, respectively. The samples were frozen promptly to the temperature $-20^{\circ} \mathrm{C}$ and stored until biochemical analysis. GCF concentrations of IL-1 $\beta$ and MMP- 8 were measured by an enzyme-linked immunosorbent assay (ELISA) using commercially available Quantikine kits (R\&D Systems, MN, USA) for quantitative assessment of total IL-1 $\beta$ and MMP-8. For MMP- 8 the test sensitivity was $0.06 \mathrm{ng} / \mathrm{ml}$, assay range $0.2-10 \mathrm{ng} / \mathrm{ml}-\beta$, whereas for IL-1 sensitivity was $1 \mathrm{pg} / \mathrm{ml}$, assay range $3.9-250 \mathrm{pg} / \mathrm{ml}$.

Statistical analyses were carried out with Statistica v. 13 (TIBCO Software Inc., Palo Alto, USA). Data were presented as mean \pm standard deviation (SD) and $95 \%$ confidence intervals. Any p values of less than $0.05(p<0.05)$ were considered statistically significant. The Mann-Whitney test was used for comparisons of two independent groups of continuous variables and the Wilcoxon signedrank test for dependent samples. Relationships between periopathogens and concentrations of MMP-8 and IL-1 were evaluated using Spearman rank correlation (R). The strength of correlations was described by $R$ value. If $\mathrm{R}$ absolute value was $>0.70$, the correlation was regarded as strong; if $\mathrm{R}$ absolute value was from 0.40 to 0.70 , the correlation was regarded as moderate; and if $\mathrm{R}$ absolute value was $<0.40$, the correlation was regarded as weak. For evaluation of multivariate relationships, multiple linear regression was applied.

Table 1. Clinical characteristics of groups (mean with 95\% confidence interval and standard deviation)

\begin{tabular}{lcccc}
\hline Variables & $\begin{array}{c}\text { Total } \\
(\boldsymbol{n}=\mathbf{4 9})\end{array}$ & $\begin{array}{c}\text { Periodontitis grade B } \\
(\boldsymbol{n}=\mathbf{3 0})\end{array}$ & $\begin{array}{c}\text { Periodontitis grade C } \\
(\boldsymbol{n}=\mathbf{1 9})\end{array}$ & $\begin{array}{c}\text { Comparison } \boldsymbol{p} \\
(\text { Mann-Whitney test })\end{array}$ \\
\hline Number of teeth & 25.55 & 25.43 & 25.74 & 0.203 \\
\hline Probing pocket depth & $2.93[2.68-3.19] \pm 0.90$ & $2.70[2.42-2.98] \pm 0.75$ & $3.31[2.82-3.79] \pm 1.00$ & 0.037 \\
(PPD) [mm] & & & & 0.023 \\
\hline$\%$ of pockets with PPD & $10.37[7.67-13.06] \pm 9.39$ & $7.70[5.36-10.04] \pm 6.25$ & $14.58[8.85-20.31] \pm 11.89$ & 0.222 \\
$\geq 6$ mm [\%] & & & & \\
\hline Full mouth plaque score & $55.53[48.74-62.33]$ & $52.47[44.09-60.84]$ & $60.37[48.17-72.57]$ & \pm 25.32 \\
(FMPI) [\%] & \pm 23.66 & \pm 22.44 & 58.121 \\
\hline $\begin{array}{l}\text { Bleeding on probing } \\
\text { (BoP) [\%] }\end{array}$ & $50.41[43.53-57.28]$ & $45.50[36.67-54.33]$ & $58.16[47.12-69.20]$ & \pm 22.90 \\
\hline
\end{tabular}




\section{Results}

The group's characteristics are presented in Table 1. Statistically significant differences were observed in relation to mean PPD and to the percentage of deep periodontal pockets depending on diagnosis. The groups did not differ in the average number of teeth and the values of FMPI and BoP.

No differences were observed in the quantitative and qualitative composition of subgingival biofilm depending on diagnosis (Table 2). Table 3 presents the number of individuals in whom particular species of periopathogens were identified.
The concentration of MMP-8 in GCF was significantly higher in the case of periodontitis Grade C (Table 4). In the multiple regression analysis, it was shown that age, gender, number of teeth, PPD mean value, PPD maximum value, percentage of deep periodontal pockets, FMPI and BoP values had no significant effect on MMP-8 concentration (Table 5). The concentration of IL-1 $\beta$ was similar in both groups.

There were no significant correlations between the occurrence of individual periopathogens and concentrations of MMP- 8 and IL-1 $\beta$ depending on diagnosis (Table 6 and Table 7).

Table 2. Microbiological characteristics of study groups with respect to diagnosis (mean with $95 \%$ confidence interval and standard deviation)

\begin{tabular}{|c|c|c|c|c|}
\hline Variables & $\begin{array}{c}\text { Total } \\
(n=49)\end{array}$ & $\begin{array}{c}\text { Periodontitis grade B ( } n \\
=\mathbf{3 0})\end{array}$ & $\begin{array}{l}\text { Periodontitis grade } C \\
\qquad(n=19)\end{array}$ & $\begin{array}{c}\text { Comparison } p \\
\text { (Mann-Whitney test) }\end{array}$ \\
\hline $\begin{array}{l}\text { Total number } \\
\text { of periopathogens }\end{array}$ & $13664980 \pm 20096741 \times 10^{3}$ & $11212133 \pm 15751879 \times 10^{3}$ & $17537895 \pm 25522516 \times 10^{3}$ & 0.790 \\
\hline $\begin{array}{l}\text { Number of } \\
\text { Porphyromonas gingivalis }\end{array}$ & $38155 \pm 101180 \times 10^{3}$ & $55141 \pm 125558 \times 10^{3}$ & $11335 \pm 25709 \times 10^{3}$ & 0.140 \\
\hline $\begin{array}{l}\% \text { Porphyromonas } \\
\text { gingivalis }\end{array}$ & $1.13 \pm 4.18$ & $0.91 \pm 2.8$ & $1.49 \pm 5.81$ & 0.105 \\
\hline $\begin{array}{l}\text { Number of Treponema } \\
\text { denticola }\end{array}$ & $18706 \pm 40508 \times 10^{3}$ & $22386 \pm 49973 \times 10^{3}$ & $12895 \pm 17151 \times 10^{3}$ & 0.967 \\
\hline$\%$ Treponema denticola & $0.25 \pm 0.44$ & $0.24 \pm 0.46)$ & $0.25 \pm 0.42$ & 0.926 \\
\hline $\begin{array}{l}\text { Number of Tannerella } \\
\text { forsythia }\end{array}$ & $13367 \pm 26112 \times 10^{3}$ & $10529 \pm 25838 \times 10^{3}$ & $17847 \pm 26608 \times 10^{3}$ & 0.140 \\
\hline$\%$ Tannerella forsythia & $0.2 \pm 0.6$ & $0.08 \pm 0.16$ & $0.38 \pm 0.92$ & 0.109 \\
\hline $\begin{array}{l}\text { Number of Prevotella } \\
\text { intermedia }\end{array}$ & $63096 \pm 207303 \times 10^{3}$ & $98761 \pm 259907 \times 10^{3}$ & $6783 \pm 17255 \times 10^{3}$ & 0.214 \\
\hline$\%$ Prevotella intermedia & $0.39 \pm 0.78$ & $0.52 \pm 0.88$ & $0.18 \pm 0.54$ & 0.157 \\
\hline $\begin{array}{l}\text { Number of Peptoscrep. } \\
\text { micros }\end{array}$ & $8490 \pm 17616 \times 10^{3}$ & $5414 \pm 10451 \times 10^{3}$ & $13347 \pm 24716 \times 10^{3}$ & 0.121 \\
\hline \% Peptoscrep. micros & $15.26 \pm 105.69$ & $24.83 \pm 135.08$ & $0.15 \pm 0.27$ & 0.169 \\
\hline $\begin{array}{l}\text { Number of Fusobacterium } \\
\text { nucleatum }\end{array}$ & $19913 \pm 74011 \times 10^{3}$ & $14837 \pm 69148 \times 10^{3}$ & $27928 \pm 82419 \times 10^{3}$ & 0.083 \\
\hline $\begin{array}{l}\% \text { Fusobacterium } \\
\text { nucleatum }\end{array}$ & $0.19 \pm 0.47$ & $0.14 \pm 0.45$ & $0.27 \pm 0.51$ & 0.062 \\
\hline $\begin{array}{l}\text { Number of Eubacterium } \\
\text { nodatum }\end{array}$ & $178 \pm 529 \times 10^{3}$ & $204 \pm 633 \times 10^{3}$ & $136 \pm 310 \times 10^{3}$ & 0.531 \\
\hline$\%$ Eubacterium nodatum & $0.00 \pm 0.00$ & $0.00 \pm 0.00$ & $0 \pm 0.01$ & 0.813 \\
\hline $\begin{array}{l}\text { Number of } \\
\text { Capnocytophaga } \\
\text { gingivalis }\end{array}$ & $12349 \pm 22592 \times 10^{3}$ & $15471 \pm 27066 \times 10^{3}$ & $7418 \pm 11789 \times 10^{3}$ & 0.485 \\
\hline $\begin{array}{l}\% \text { Capnocytophaga } \\
\text { gingivalis }\end{array}$ & $0.18 \pm 0.28$ & $0.24 \pm 0.33$ & $0.09 \pm 0.13$ & 0.246 \\
\hline $\begin{array}{l}\text { Number of } A . \text { actinomy- } \\
\text { cetemcomitans }\end{array}$ & $1887 \pm 12711 \times 10^{3}$ & $19 \pm 75 \times 10^{3}$ & $4837 \pm 20392 \times 10^{3}$ & 0.935 \\
\hline $\begin{array}{l}\% \text { A. actinomycetem- } \\
\text { comitans }\end{array}$ & $0.00 \pm 0.02$ & $0.00 \pm 0.00$ & $0.01 \pm 0.03$ & 0.766 \\
\hline
\end{tabular}


Table 3. Number of subjects in whom specific periopathogens were found

\begin{tabular}{lccc}
\hline Variables & $\begin{array}{c}\text { Total } \\
(\boldsymbol{n}=\mathbf{4 9})\end{array}$ & $\begin{array}{c}\text { Periodontitis } \\
\text { grade B } \\
(\boldsymbol{n}=\mathbf{3 0})\end{array}$ & $\begin{array}{c}\text { Periodontitis } \\
\text { grade C } \\
(\boldsymbol{n}=\mathbf{1 9})\end{array}$ \\
\hline $\begin{array}{l}\text { Porphyromonas } \\
\text { gingivalis }\end{array}$ & 28 & 19 & 9 \\
\hline Treponema denticola & 34 & 21 & 13 \\
\hline Tannerella forsythia & 31 & 18 & 13 \\
\hline $\begin{array}{l}\text { Prevotella intermedia } \\
\text { Peptoscrep. micros }\end{array}$ & 22 & 15 & 7 \\
\hline Fusobacterium nucleatum & 28 & 15 & 13 \\
\hline Eubacterium nodatum & 9 & 4 & 5 \\
\hline $\begin{array}{l}\text { Capnocytophaga } \\
\text { gingivalis }\end{array}$ & 40 & 24 & 16 \\
\hline A. actinomycetemcomitans & 5 & 3 & 2 \\
\hline
\end{tabular}

Table 5. Correlations between MMP- 8 crevicular fluid with respect to selected general and local patient-related factors

\begin{tabular}{lcc}
\hline Variables & $\boldsymbol{b}$ & $\boldsymbol{p}$ \\
\hline Age & 0.022 & 0.914 \\
\hline Sex & 0.253 & 0.172 \\
\hline Number of teeth & -0.308 & 0.161 \\
\hline FMPI & -0.043 & 0.854 \\
\hline BoP & 0.348 & 0.188 \\
\hline PPD max & 0.032 & 0.882 \\
\hline PPD mean & -0.369 & 0.225 \\
\hline$\%$ PPD $\geq 6$ mm & 0.150 & 0.626 \\
\hline b- standard regression coefficient &
\end{tabular}

Table 4. Concentrations of MMP- 8 and IL-1 $\beta$ in gingival crevicular fluid with respect to diagnosis (mean \pm standard deviation)

\begin{tabular}{|c|c|c|c|c|}
\hline Variables & $\begin{array}{c}\text { Total } \\
(n=49)\end{array}$ & $\begin{array}{c}\text { Periodontitis grade B } \\
\quad(\boldsymbol{n}=\mathbf{3 0})\end{array}$ & $\begin{array}{l}\text { Periodontitis grade } C \\
\quad(n=19)\end{array}$ & $\begin{array}{c}\text { Comparison } p \\
\text { (Mann-Whitney test) }\end{array}$ \\
\hline 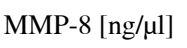 & $46 \pm 39$ & $37 \pm 28$ & $61 \pm 49$ & 0.039 \\
\hline IL-1 $\beta[\mathrm{pg} / \mu \mathrm{l}]$ & $64 \pm 41$ & $65 \pm 47$ & $63 \pm 33$ & 0.782 \\
\hline
\end{tabular}

\section{Discussion}

There are still no clearly defined markers that could facilitate risk assessment of periodontitis occurrence and then the dynamics of its course. In our study we applied highly sophisticated and reproducible technologies that allowed us to use minimal amounts of biofilm and gingival crevicular fluid to examine microbiologic and host factors simultaneously. For this purpose, the authors carried out a thorough microbiological analysis of the biofilm from deep periodontal pockets and an assessment of concentration of two inflammatory mediators from GCF. Two groups were selected - the first group consisted of 30 patients with advanced periodontitis with mild, chronic course (Grade B periodontitis), the second group comprised 19 patients with advanced periodontitis with dynamic, aggressive course (Grade $\mathrm{C}$ periodontitis). There were no significant differences in the microbiological profile of the pockets, whereas in patients with periodontitis Grade C, a significantly higher concentration of MMP-8 in GCF was observed, which may indicate an increased non-specific immune-inflammatory response to plaque microbiota in this group of patients. There were no significant correlations between the occurrence of individual periopathogens and concentrations of the assessed biomarkers in GCF depending on diagnosis.
For many years, specific diseases and their course have been attributed to specific pathogens of the microbiota [16]. Particular attention was paid to the microbiota of the red complex and A. actinomycetemcomitans [4]. A correlation was found between a large number $\left(>10^{5}\right.$ bacterial cells in a sample) of the red complex bacteria and the advancement of periodontitis [17]. Presence of specific periopathogens in the biofilm (e.g. A. actinomycetemcomitans) was associated with the progression of the disease manifested by rapid connective tissue attachment loss. There was a special relationship between the presence of highly leukotoxic strains of A. actinomycetemcomitans and typical clinical symptoms of the localized form of aggressive periodontitis in first molars and incisors [18]. However, the presence and distribution of individual types of microbiota did not always correlate with the way that periodontitis started and progressed, hence specific plaque theory was revised [17]. It has been proven that microbiota in deep periodontal pockets do not necessarily cause their formation, and only the environment existing in such pockets was conducive to their colonization by microbiota strains [19]. Nibali et al. [20] evaluated the presence of A. actinomycetemcomitans and $P$. gingivalis in 267 patients diagnosed with chronic or aggressive periodontitis. A. actinomycetemcomitans was present in $54 \%$ of patients with aggressive periodontitis and in $48 \%$ of patients with 
chronic periodontitis. The presence of $P$. gingivalis was detected more frequently - but only slightly - in patients with chronic periodontitis $(67 \%)$ compared to patients with aggressive periodontitis (52\%). In another study, $47.50 \%$ of samples tested positive for A. actinomycetemcomitans in aggressive periodontitis group and $42.50 \%$ of samples in chronic periodontitis group [21]. Moreover, some reports indicated that $P$. gingivalis might play a double role as normal flora and as a pathogen, since it may constitute an ingredient of subgingival biofilm in subjects with healthy periodontium, as well as behave as a commensal opportunistic pathogen [22]. By the same token, some strains of A. actinomycetemcomitans are recognized as opportunistic pathogens whereas specific JP2 clone has attributes of a true exogenous pathogen [23]. Quite similarly, the result of our study does not provide sustenance for the assumption, that qualitative and quantitative composition of subgingival biofilm may be directly related to periodontitis form. Taking everything into consideration, we may hypothesize that periodontitis grade might not be discriminated according to a simple analysis of subgingival plaque alone. However, we speculate that periopathogens may modulate host immunologic system and pave the way for either slower or faster pattern of periodontal tissue destruction and disease progression.

Although periopathogens found in deep pockets have a damaging potential, they are insignificant compared to harmful effects resulting from the host's reactions. In this way, the balance between microbiota activity and defense processes is violated. The above mentioned nonspecific defense response of the host appears to be the major factor influencing periodontal tissue destruction. The effectiveness of the host response may be an important determinant of susceptibility to formation and unfavorable clinical course of periodontitis. Generally speaking, migration of neutrophils stimulated by periopathogens and macrophages to inflammatory sites, brings about increased concentration of proinflammatory mediators. What is more, cytokines secreted by these cells successively trigger stationary cells to produce other bioactive molecules. PMN leukocytes stimulated in vitro secreted MMP-8 and MMP-9 from intracellular granules within a few seconds after stimulus [24]. Cytokines form an intricate network that has relevance to the equilibrium between host protection and destruction. An analysis of concentrations of the main products of non-specific immune response in GCF might be useful while evaluating the severity and dynamics of innate immune responses, as they might differ among patients with periodontitis Grade B or Grade C. Moreover, recent systematic reviews concluded that neutrophilic polymorphonuclear leukocytes might play a crucial role in periodontal disease pathogenesis [25]. Numerous studies reported significantly higher concentrations of IL-1 $\beta$ and MMP- 8 in GCF in patients with diagnosed periodontitis, as compared to healthy subjects [26-35]. The majority of reports stated that IL-1 levels were higher in deep pockets, as compared to shallow pockets [27, 32, 33]. However, some authors did not notice such relationship [30, 34]. While all of the abovementioned studies described patients with chronic periodontitis, only few studies investigated GCF biomarkers in patients with different periodontal diseases [29, 31, 34]. Nędzi-Góra et al. [34] evaluated concentrations of IL-1 $\beta$ and MMP-8 in GCF in patients with advanced chronic and aggressive periodontitis and observed no difference in concentrations of the tested mediators with reference to diagnosis. Quite similarly in a study by Becerik et al. [29] GCF levels of IL-1 $\beta$, IL-6 and IL-11 were not significantly different among study groups. Comparing these observations to the results of our study, we can attribute the obtained measurements to the particular composition of the study group. The elevated concentrations of MMP-8 in patients with periodontitis Grade $\mathrm{C}$ in our study might reflect increased activity of immunocompetent cells and more severe non-specific immunological reaction, as compared to patients with periodontitis Grade B. However, based on recent comprehensive review that analyzed the profile of cytokines/chemokines in GCF, the current weight of evidence seems to be insufficient to prove major immunological differences between aggressive and chronic periodontitis [36].

There are some limitations to our study that need to be underlined. First, samples were collected from only 49 patients, including 19 Grade $\mathrm{C}$ periodontitis patients. This sample is relatively small and the sizes of both groups are different. A diagnosis was made based on clinical and radiological examination, and not on observation of patients over several years, which would be the optimal procedure in assessing the dynamics and rate of disease progression. It seems very important to continue similar studies based on a larger number of patients. Second, we evaluated only concentrations of IL- $1 \beta$ and MMP- 8 in GCF as potential biomarkers of periodontitis, hence the importance of other cytokines and bioactive agents in clinical classification and disease progression is still to be established. Interesting conclusions could be provided by observation of patients over time and by another microbiological and biochemical analysis, which would allow even better correlation of the assessed variables with the progression of periodontitis. The role of periodontal treatment should also be taken into account both with respect to changes in the quantitative and qualitative composition of subgingival biofilm and biomarkers in GCF, as well as to changes in the risk profile of periodontal disease progression.

\section{Conclusions}

In the light of the results of our study, we may speculate that periodontitis grade may not be distinguished according to a microbial analysis of subgingival biofilm or to the level of IL-1 $\beta$ in GCF. On the other hand, the higher 
concentrations of MMP-9 in GCF from deep pockets may be helpful in predicting a dynamic and more aggressive pattern of tissue destruction in the course of periodontitis. It should be expected that in future diagnostics of periodontitis, the most important role will be played by tests that analyze the presence of cytokines and inflammatory mediators in a qualitative and quantitative manner. These technologies will allow significant progress in prevention, diagnosis, and treatment of periodontal diseases, especially those with rapid pattern of progression.

The authors declare no conflict of interest.

\section{References}

1. Page RC, Kornman KS (1997): The pathogenesis of human periodontitis: an introduction. Periodontol 2000 14: 9-11.

2. Tonetti MS, Greenwell H, Kornman KS (2018): Staging and grading of periodontitis: Framework and proposal of a new classification and case definition. J Clin Periodontol 45 (Suppl 20): 149-161.

3. Loos BG, Papantonopoulos G, Jepsen S, et al. (2015): What is the contribution of genetics to periodontal risk? Dent Clin North Am 59: 761-780.

4. Socransky SS, Haffajee AD (1992): Microbial mechanisms in the pathogenesis of destructive periodontal diseases: current concepts. J Periodontol 63: 322-331.

5. Stobernack T, Teil Espina M, Mulder LM, et al. (2018): A secreted bacterial peptidylarginine deiminase can neutralize human innate immune defenses. mBio 9: pii: e01701-18.

6. Barth K, Remick DG, Genco CA (2013): Disruption of immune regulation by microbial pathogenes and resulting chronic inflammation. J Cell Physiol 228: 1413-1422.

7. Asai T, Okamoto-Shinayama K, Kikuchi Y, et al. (2018): Characterization of a novel potential peptide import system in Treponema denticola. Microb Pathog 123: 467-472.

8. Aberg HC, Kelk P, Johansson A (2015): Aggregatibacter actinomycetemcomitans: virulence of its leukotoxin and association with aggressive periodontitis. Virulence 6: 188-195.

9. Rajakaruna GA, Negi M, Uchida K, et al. (2018): Localization and density of Porphyromonas gingivalis and Tannerella forsythia in gingival and subgingival granulation tissues affected by chronic or aggressive periodontitis. Sci Rep 8: 9507.

10. Zhu H, Lu S, Wei M, et al. (2018): Identification of novel genes involved in gingival epithelial cells respnding to $\mathrm{Ag}$ gregatibacter actinomycetemcomitans and Porphromonas gingivalis infections. Arch Oral Biol 96: 113-121.

11. Page RC (1991): The role of inflammatory mediators in the pathogenesis of periodontal disease. J Periodontal Res 26 (3 Pt 2): 230-242.

12. Ruscitti P, Cipriani P, Carubbi F, et al. (2015): The role of $\mathrm{Il}-1 \beta$ in the bone loss during rheumatic diseases. Mediators Inflamm 2015: 782382.

13. Palomo J, Dietrich D, Martin P, et al. (2015): The interleukin (IL)-1 cytokine family- balance between agonists and antagonists in inflammatory diseases. Cytokine 76: 25-37.

14. O'Leary TJ, Drake RB, Naylor JE (1972): The plaque control record. J Periodontol 43: 38-46.

15. Ainamo J, Bay I (1975): Problems and proposal for recording gingivitis and plaque. Int Dent J 25: 229-235.
16. Theilade E (1986): The non-specific theory in microbial etiology of inflammatory periodontal diseases. J Clin Periodontol 13: 905-911.

17. Papapanou PN (2002): Population studies of microbial ecology in periodontal health and disease. Ann Periodontol 7: 54-61.

18. Stabholz A, Soskolne A, Shapira L (2010): Genetic and environmental risk factors for chronic periodontitis and aggressive periodontitis. Periodontol 2000 53: 138-153.

19. Mombelli A, Nyman S, Brăgger N, et al. (1995): Clinical and microbiological changes associated with an altered subgingival environment induced by periodontal pocket reduction. J Clin Periodontol 22: 780-787.

20. Nibali L, D'Aiuto F, Ready D, et al. (2012): No association between A. actinomycetemcomitans or P. gingivalis and chronic or aggressive periodontitis diagnosis. Quintessence Int 43: 247-254

21. Suprith SS, Setty S, Bhat K, et al. (2018): Serotypes of Aggregatibacter actinomycetemcomitans in relation to periodontal status and assessment of leukotoxin in periodontal disease: a clinico-microbiological study. J Indian Soc Periodontol 22: 201-208.

22. Elamin A, Ali RW, Bakken V (2017): Putative periodontopathic bacteria and herpes viruses interactions in the subgingival plaque of patients with aggressive periodontitis and healthy controls. Clin Exp Dent Res 3: 183-190.

23. Könönen E, Müller HP (2014): Microbiology of aggressive periodontitis. Periodontol 2000 65: 46-78.

24. Birkedal-Hansen H (1993): Role of matrix metalloproteinases in human periodontal diseases. J Periodontol 64: 474-484.

25. Cekici A, Kantarci A, Hasturk H, Van Dyke TE (2014): Inflammatory and immune pathways in the pathogenesis of periodontal disease. Periodontol 2000 64: 57-80.

26. Akbari G, Prabhuji ML, Karthikeyan BV, et al. (2015): Analysis of matrix metalloproteinase-8 levels in gingival crevicular fluid and whole mouth fluid among smokers and non-smokers using enzyme-linked immune-sorbent assay and a novel chair-side test. J Indian Soc Periodontol 19: 525-530.

27. Baser U, Oztekin G, Ademoglu E, et al. (2014): Is the severity of periodontitis related to gingival crevicular fluid and serum high-sensitivity C-reactive protein concentrations? Clin Lab 60: 1653-1658.

28. Baeza M, Garrido M, Hernández-Rios P, et al. (2016): Diagnostic accuracy for apical and chronic periodontitis biomarkers in gingival crevicular fluid: an exploratory study. J Clin Periodontol 43: 34-45.

29. Govindarajan K, Muthukumar S, Rangarao S (2015): Relationship between interleukin $1 \alpha$ levels in the gingival crevicular fluid in health and inflammatory periodontal disease and periodontal inflamed surface area: a correlative study. J Indian Soc Periodontol 19: 618-623.

30. Leppilahti JM, Sorsa T, Kallio MA, et al. (2015): The utility of gingival crevicular fluid matrix metalloproteinase- 8 response patterns in prediction of site-level clinical treatment outcome. J Periodontol 86: 777-787.

31. Lomba KS, Meiler TF, Sete MR, et al. (2015): Use of minimally invasive gingival biopsies in the study of inflammatory mediators expression and their correlation with gingival fluid in patients with chronic periodontitis. Indian J Dent Res 26: 126-130.

32. Shimada Y, Tabeta K, Sugita N, Yoshie H (2013): Profiling biomarkers in gingival crevicular fluid using multiplex bead immunoassay. Arch Oral Biol 58: 724-730. 
33. Nędzi-Góra M, Górska R, Kostrzewa-Janicka J, Kowalski J (2017): Concentration of MMP-8 and Il-1 $\beta$ in gingival crevicular fluid in patients with chronic and aggressive periodontitis. Centr Eur J Immunol 42: 342-346.

34. Victor DJ, Subramanian S, Gnana PP, Kolagani SP (2014): Assessment of matrix metalloproteinases-8 and -9 in gingival crevicular fluid of smokers and non-smokers witch chronic periodontitis using ELISA. J Int Oral Health 6: 67-71.

35. Duarte PM, Bastos MF, Fermiano D, Rabelo CC, et al. (2015): Do subjects with aggressive and chronic periodontitis exhibit a different cytokine/chemokine profile in the gingival crevicular fluid? A systematic review. J Periodontal Res 50: 18-27. 